International Journal of Diabetology \& Vascular Disease Research (IJDVR)

ISSN:2328-353X

\title{
Effect of Aqueous Extracts of some Ayurvedic Medicinal Plants on Tissues Involved in Glucose Homeostasis in vitro
}

Suresh K. Mohankumar ${ }^{1}$, James R. McFarlane $2^{2 *}$

Research Article

${ }^{1}$ Science/Biomedical Science, University of Nottingham Malaysia Campus, Semenyih, Malaysia.

${ }^{2}$ Centre for Bioactive Discovery, School of Science and Technology, University of New England, Armidale, Australia.

\section{Abstract}

Aqueous extracts of some antidiabetic Ayurvedic plant treatments such as Trigonella foenum-graecum Linn (TFG), Pterocarpus marsupium Roxb (PM), Gymnema sylvestre R.Br (GS) and Curcuma longa Linn (CL) were tested for their biological effects on glucose uptake by mouse muscle tissue and insulin secretion from mouse pancreas under both basal and hyperglycemic culture conditions. The results indicate that none of these treatments have a significant effect on either glucose uptake by muscle or insulin secretion from the pancreas under basal conditions. Under hyperglycemic conditions all treatments except GS demonstrate significant effects on glucose uptake by muscle tissue, and all treatments were effective at increasing insulin secretion from pancreas. This suggests that all these plant treatments enhance glucose homeostasis by stimulating either insulin secretion (GS) or enhancing glucose uptake (TFG) or activating both (CL and PM).

Keywords: Ayurvedic Medicinal Plants; Diabetes; Insulin Secretion; Glucose Uptake.

\author{
*Corresponding Author: \\ James R. McFarlane, \\ Centre for Bioactive Discovery, School of Science and Technology, \\ University of New England, Armidale, NSW 2351, Australia. \\ E-mail: jmcfarla@metz.une.edu.au
}

Received: May 14, 2015

Accepted: June 20, 2015

Published: July 01, 2015

Citation: Suresh K. Mohankumar, James R. McFarlane (2015) Effect of Aqueous Extracts of some Ayurvedic Medicinal Plants on Tissues involved in Glucose Homeostasis in vitro. Int J Diabetol Vasc Dis Res, 3(6) 105-107. doi: http://dx.doi.org/10.19070/2328-353X-1500021

Copyright: James R. McFarlane ${ }^{\mathcal{O}}$ 2015. This is an open-access article distributed under the terms of the Creative Commons Attribution License, which permits unrestricted use, distribution and reproduction in any medium, provided the original author and source are credited.

\section{Introduction}

Incidence of Type 2 diabetes (T2D) is increasing worldwide. A number of undesirable side effects are associated with current conventional treatments and they are not always effective in maintaining euglycemia or preventing later complications associated with the disease. Consequently the search for new antidiabetic compounds is intense. Ayurveda a traditional Indian system of medicine advocates a wide range of medicinal plants to treat T2D. Plants such as Trigonella foenum-graecum Linn, Pterocarpus marsupium Roxb, Gymnema sylvestre R.Br and Curcuma longa Linn, either individually or in combination have been traditionally used as an Ayurvedic remedy in T2D and related complications [1]. The antidiabetic effect of these plants has been studied in animals [2-6] as well as in humans [7-9]. Despite the numerous studies that have demonstrated that these plants reduce blood glucose levels in a number of species including humans, the biological mechanisms, major site of action and active ingredients for these hypoglycemic effects are not known. Hence, the present study aimed to investigate the effect of aqueous extracts of these plant treatments on glucose uptake by mouse muscle tissues and also insulin secretion from mouse pancreas under normal and hyperglycemic conditions in vitro.

\section{Materials and Methods}

\section{Plant material preparation}

Gymnema sylvestre R.Br. (Apocynaceae) leaves (GS) and Curcuma longa Linn. (Zingiberaceae), rhizome (CL) were purchased from Austral herbs, Uralla, NSW 2358, Australia. Pterocarpus marsupium Roxb. (Febaceae), hardwood (PM) was directly imported from India. Trigonella foenum-graecum Linn. (Febaceae), seeds (TFG) were purchased from local commercial health foods market, Armidale, NSW 2350, Australia. GS, CL and TFG extracts were prepared by soaking $100 \mathrm{~g}$ of the material in 1 litre of water at ambient temperature overnight. Aqueous extracts of heartwood of PM was prepared by soaking one block (30-35gm) of PM in 10 litre of water at ambient temperature for 10 days. The extracts obtained were then filtered (Whatman no.1) to remove any particles and the filtrate was further centrifuged at $5000 \mathrm{rpm}$ for $15 \mathrm{~min}$ to remove fine particles and stored at $-20^{\circ} \mathrm{C}$. Immediately before use the extracts were sterilized through a $0.22 \mu \mathrm{m}$ syringe filter (Sarstedt Australia Pty Limited, South Australia 5095).

\section{Animals and tissue preparation}

Male Swiss mice of adult age, weighing approximately 18-22g were obtained from the physiology mouse colony at the University of New England, Armidale, NSW 2351, Australia. They were euthanased via $\mathrm{CO}_{2}$ asphyxiation. The pancreas and the skeletal 
muscle from the abdomen were removed and placed on ice in phosphate buffered saline (PBS) of a neutral $\mathrm{pH}$. Muscle and pancreas tissues were dissected in to strips approximately $5 \mathrm{~mm}$ long, $2 \mathrm{~mm}$ wide, $2 \mathrm{~mm}$ thick and $2 \mathrm{~mm}$ long, $2 \mathrm{~mm}$ wide, $2 \mathrm{~mm}$ thick respectively and rinsed with PBS immediately before the incubation. All of the animal experimentation was approved by University of New England Animal ethics committee and are in accordance with NH \& MRC guidelines for animal experimentations. All the experiments were carried out in quadruplicates and repeated once.

\section{Tissue culture bioassay}

Pancreas or muscle tissues ( 5 pieces/well) were separately transferred to the 24 well tissue culture plates containing $1 \mathrm{ml}$ of pre sterilized Dulbecco's modified Eagle's medium (Invitrogen Australia Pty Ltd, Australia) through $0.45 \mu \mathrm{m}$ syringe filters, supplemented with $0.1 \%$ bovine serum albumin (Sigma Aldrich Pty Ltd, Australia) and $0.01 \%$ antibiotic and antimycotic solution (Invitrogen Australia Pty Ltd, Australia). Glucose concentration in the media was $5 \mathrm{mmol} / \mathrm{L}$ or $12 \mathrm{mmol} / \mathrm{L}$ in order to mimic normal and hyperglycemic culture conditions respectively. Incubation were performed in a humidified atmosphere of $5 \% \mathrm{CO}_{2}$ in air at $37^{\circ} \mathrm{C}$ for 24 hours, with the treatments $(10 \mu \mathrm{l})$ is compared with saline $(0.9 \% \mathrm{NaCl})$ as a control, tolbutamide- $0.1 \mathrm{mmol} / \mathrm{L}$ and metformin - $2 \mathrm{mmol} / \mathrm{L}$ (Sigma Aldrich Pty Ltd, Australia) as a positive control in pancreas and muscle culture respectively. Immediately after the experiment, media and tissue samples were stored at $-20^{\circ} \mathrm{C}$ until further examined. All the doses of treatments and positive control were derived from respective preliminary dose response curve experiments (data not shown). A dose that represents a maximal efficacy was chosen and used in this study.

\section{Glucose analysis}

The frozen culture media samples were thawed at room temperature and $100 \mu \mathrm{l}$ of muscle culture media samples was analyzed using DADE clinical analyzer (DADE-XL, Dupont, USA) for glucose content. The glucose method was an adaptation of the hexokinase-glucose-6-phosphate dehydrogenase method and this method is more specific than general reducing sugar methods [10]. Glucose uptake was calculated based on the difference in glucose concentration between control wells with and without tissues.

\section{Insulin assay}

Pancreas culture media insulin concentration was determined by radio-immuno assay (RIA). Insulin concentration of the pancreas culture media samples were calculated in $\mu \mathrm{IU} / \mathrm{ml}$ using a linear equation derived from $\log / \operatorname{logit}$ transformation of the RIA standard curve. Briefly, $100 \mu$ l of culture media samples and standards (in DMEM) were added to RIA tubes in duplicate followed by the addition of $100 \mu \mathrm{l}$ of anti-bovine insulin $(1: 8000)$ and $100 \mu$ l of radioactive insulin tracer $(20,000 \mathrm{cpm} / 100 \mu \mathrm{l})$. All these tubes vortexed and incubated at $4{ }^{\circ} \mathrm{C}$ overnight. After overnight incubation $1.1 \mathrm{ml}$ of $20 \%$ polyethylene glycol (PEG) and $50 \mu \mathrm{l}$ of charcoal striped horse serum were added and further incubated for 15-20 min at room temperature then centrifuged at $3000 \mathrm{rpm}$ for 30 minutes followed by aspiration. The pellet containing the bound fraction was immediately counted for $\gamma$-decay using an automatic Gamma counter (Wallac, 1470 WIZARD).

\section{Statistical analysis}

Experimental data were analyzed statistically, using the general linear model procedure in SAS statistical software (SAS Institute Inc. Cary, NC, USA). The data were evaluated using 2-way ANOVA followed by Student-Newman Keuls post hoc test. Values were considered to be significantly different at $\mathrm{p}<0.05$ and presented as mean \pm standard error.

\section{Results}

The effects of TFG, CL, GS and PM treatments on glucose uptake and on insulin secretion under hyperglycemic conditions have been converted as folds of control and presented in Table 1.

\section{Muscle glucose uptake}

There was no significant difference in muscle glucose uptake under basal culture conditions between the control and the extracts ( $p>0.05)$. Under hyperglycemic conditions plant treatments, CL, TFG and PM, significantly increased the glucose uptake activity ( $p>0.05$ ). However, under similar experimental conditions, GS failed to produce a significant glucose uptake activity. Treatment with CL extract showed a peak glucose uptake activity among other plant treatments and this effect was significantly different from the control and also directly comparable with the effect of

Table 1. Effects of some Ayurvedic plant treatments on tissues involved in glucose homeostasis in vitro.

\begin{tabular}{|c|c|c|c|c|}
\hline \multirow{2}{*}{ Treatment } & \multicolumn{2}{|c|}{ Glucose uptake in folds of control } & \multicolumn{2}{c|}{ Insulin in folds of control } \\
\cline { 2 - 5 } & Basal & Hyperglycemic & Basal & Hyperglycemic \\
\hline Control & $1.00 \pm 0.07^{\mathrm{A}}$ & $1.02 \pm 0.02^{\mathrm{A}}$ & $1.00 \pm 0.05^{\mathrm{AB}}$ & $1.00 \pm 0.06^{\mathrm{A}}$ \\
\hline TFG & $0.97 \pm 0.07^{\mathrm{A}}$ & $1.54 \pm 0.03^{\mathrm{C}}$ & $1.00 \pm 1.31^{\mathrm{AB}}$ & $2.05 \pm 0.14^{\mathrm{B}}$ \\
\hline GS & $0.84 \pm 0.07^{\mathrm{A}}$ & $0.96 \pm 0.05^{\mathrm{A}}$ & $0.94 \pm 0.03^{\mathrm{A}}$ & $2.44 \pm 0.15^{\mathrm{C}}$ \\
\hline CL & $0.98 \pm 0.06^{\mathrm{A}}$ & $1.66 \pm 0.11^{\mathrm{C}}$ & $1.00 \pm 0.11^{\mathrm{AB}}$ & $2.54 \pm 0.16^{\mathrm{C}}$ \\
\hline PM & $0.97 \pm 0.06^{\mathrm{A}}$ & $1.26 \pm 0.07^{\mathrm{B}}$ & $1.04 \pm 0.05^{\mathrm{AB}}$ & $3.76 \pm 0.42^{\mathrm{D}}$ \\
\hline Metformin & $1.08 \pm 0.03^{\mathrm{A}}$ & $1.65 \pm 0.18^{\mathrm{C}}$ & - & - \\
\hline Tolbutamide & - & - & $1.11 \pm 0.06^{\mathrm{B}}$ & $3.36 \pm 0.15^{\mathrm{E}}$ \\
\hline
\end{tabular}

Data are expressed in mean \pm standard error

Means without a common letter are significantly different $(\mathrm{p}<0.05)$ 
metformin

\section{Pancreas insulin secretion}

Under basal culture conditions, all the treatments showed no significant difference from control in stimulating insulin secretion from pancreas $(p>0.05)$. However, all treatments under hyperglycemic conditions showed a significant increase in the media insulin and they were significantly different from the control $(p$ $<0.05)$. The maximal media insulin was observed with the PM treatment and it was significantly greater than the media insulin of tolbutamide treatment $(\mathrm{p}<0.05)$.

\section{Discussion}

The present study for the first time reports the effects of these treatments on insulin secretion and action under both and basal and hyperglycemic culture conditions. Glucose uptake under basal and non-basal culture conditions has been reported to be the one of the most sensitive indicators of in vitro functional viability of the tissues [11]. The results of glucose uptake from the present experiments, compares favorably with earlier reports in the literature, and moreover significant response of the tissues when treated with metformin substantiates that the tissue culture protocol is functional as well as metabolically viable $[12,13]$. Similarly, the significant response of pancreas with tolbutamide and also their significant stimulatory response from basal to hyperglycemic conditions demonstrate that the in vitro assay technique is metabolically functional and reproducible.

Of these plant treatments, TFG, CL and PM showed a significant glucose uptake activity, suggesting these plant treatments may have either protective effects on tissues to keep them metabolically viable under hyperglycemic conditions or mimic insulin action or act via insulin-mediated enhanced peripheral glucose uptake like metformin [14]. However the GS treatment failed to produce enhanced activity under similar conditions, which supports the earlier findings, where it has been suggested that GS extract have no effect on peripheral glucose uptake [15].

Insulin secretion by pancreatic $\beta$-cells in response to glycemic control is maintained by glucose, other nutrients, neurotransmitters and other hormones [16]. However glucose is the primary regulator of insulin synthesis and secretion. The enhanced insulin secreting activity of plant treatments in response to glucose, suggest that these treatments have either protective or regenerative activity on $\beta$-cell metabolism that leading to insulin secretion.

\section{Conclusion}

In conclusion, all these plant treatment enhance glucose homeostasis by increasing either insulin secretion (GS) or peripheral glucose uptake (TFG) or both (CL and PM). The findings of antidiabetic properties in tissue culture-based bioassay support the ethnopharmacological use of these Ayurvedic plant and also indicates the possible mechanistic ways in which they are achieving glucose lowering effects. Moreover, the in vitro model and methods described in this study could be used for the development of new antidiabetic drugs. Nonetheless, these promising in vitro results must be ascertained in various in vitro and in vivo animal models of insulin resistance and diabetes.

\section{References}

[1]. Grover JK, Yadav S, Vats V (2002) Medicinal plants of India with anti-diabetic potential. J Ethnopharmacol 81(1): 81-100.

[2]. Arun N, Nalini N (2002) Efficacy of turmeric on blood sugar and polyol pathway in diabetic albino rats. Plant Foods Hum Nutr 57(1): 41-52.

[3]. Khosla P, Gupta DD, Nagpal RK (1995) Effect of Trigonella foenum graecum (fenugreek) on blood glucose in normal and diabetic rats. Indian J Physiol Pharmacol 39(2): 173-174.

[4]. Kuroda M, Mimaki Y, Nishiyama T, Mae T, Kishida H, et al. (2005) Hypoglycemic effects of turmeric (Curcuma longa L. rhizomes) on genetically diabetic KK-Ay mice. Biol Pharm Bull 28(5): 937-939.

[5]. Persaud SJ, Al-Majed H, Raman A, Jones PM (1999) Gymnema sylvestre stimulates insulin release in vitro by increased membrane permeability. J Endocrinol 163(2): 207-212.

[6]. Saifi AQ, Shinde S, Kavishwar WK, Gupta SR (1971) Some aspects of phytochemistry and hypoglycemic actions of Pterocarpus marsupium. J Res Indian Med 6: 205-207.

[7]. Baskaran K, Ahamath BK, Shanmugasundaram KR, Shanmugasundaram ER (1990) Antidiabetic effect of a leaf extract from Gymnema sylvestre in non-insulin-dependent diabetes mellitus patients. J Ethnopharmacol 30(3): 295-305.

[8]. Rajasekharan S, Tuli SN (1976) Pterocarpus marsupium, in the treatment of madhumeha (diabetes mellitus) - A clinical trial. J Res Indian Med Yoga Homeo 11: 9-14.

[9]. Sharma RD, Sarkar A, Hazra DK, Mishra B, Singh JB, et al. (1996) Use of Fenugreek seed powder in the management of non-insulin dependent diabetes mellitus. Nutrition Research 16(8): 1331-1339.

[10]. Kunst A, Draeger B, Ziegenhorn J (1983) UV-methods with hexokinase and glucose-6-phosphate dehydrogenase. Methods of enzymatic analysis 6: 163172.

[11]. Dohm GL, Tapscott E, Pories WJ, Dabbs DJ, Flickinger EG, et al. (1988) An in vitro human muscle preparation suitable for metabolic studies. Decreased Insulin stimulation of glucose transport in muscle from morbidity obese and diabetic subjects. J Clin Invest 82(2): 486-494.

[12]. Hundal HS, Ramlal T, Reyes R, Leiter L, Klip A (1992) Cellular mechanism of metformin action involves glucose transporter translocation from an intracellular pool to the plasma membrane in L6 muscle cells. Endocrinology 131(3): 1165-1173.

[13]. Tsiani E, Ramlal T, Leiter L, Klip A, Fantus IG (1995) Stimulation of glucose uptake and increased plasma membrane content of glucose transporters in L6 skeletal muscle cells by sulphonylureas and gliclazide and glyburide. Endocrinology 136(6): 2505-2512.

[14]. Matthaei S, Hamann A, Klein H, Benecke HH, Kreymann G, et al. (1991) Association of metformin's effect to increase insulin-simulated glucose transport with potentiation of insulin-induced translocation of glucose transporters from intracellular pool to plasma membrane in rat adipocytes. Diabetes 40: 850-857.

[15]. Shanmugasundaram ER, Rajeswari G, Baskaran K, Kumar BRR, Shanmugasundaram KR, et al. (1990) Use of Gymnema sylvestre leaf extract in the control of blood glucose in insulin-dependent diabetes mellitus. J Ethnopharmacol 30(3): 281-294.

[16]. Wollheim CB, Lang J, Regazzi R (1996) The exocytotic process of insulin secretion and its regulation by $\mathrm{Ca}^{+2}$ and G-proteins. Diabetes review 4: 276297. 